\title{
Innovative Methodologies for Exploring the Future of Automated Vehicle Guidance
}

\author{
V. A. W. J. MARCHAU ${ }^{1 *}$ AND R. E. C. M. VAN DER \\ HEIJDEN ${ }^{2}$ \\ ${ }^{1}$ Faculty of Technology, Policy and Management, Delft University \\ of Technology, The Netherlands \\ ${ }^{2}$ Nijmegen School of Management, Nijmegen University, \\ The Netherlands
}

\begin{abstract}
In various countries, transport policy makers are increasingly interested in the automation of vehicle driving tasks. Current policy developments regarding Automated Vehicle Guidance (AVG) are complicated by several uncertainties about the development of AVG technologies, whether their implementation will contribute to or conflict with transport policy goals, and the basic societal conditions that are required for AVG implementation. In this article, we present an approach to reduce the uncertainty regarding alternative AVG implementations. In particular, we develop a backcasting approach to limit the scope of policy development and research by eliminating parts of the large variety in possible AVG developments. This approach consists of the following steps: (1) the specification of plausible AVG concepts; (2) the analysis of the conditions for the implementation of these concepts, resulting in a set of promising AVG concepts and (3) the analysis whether stakeholders' decisions and actions related to the implementation of plausible concepts will be fulfilled in time. This approach helps to eliminate non-plausible, non-promising and non-accepted AVG concepts and reduce the scope of policy making to the most viable ones. Copyright (C) 2003 John Wiley \& Sons, Ltd.
\end{abstract}

KEY WORDS transport policy; automated driving; technology assessment

\section{INTRODUCTION}

Modern societies are increasingly confronted with the road traffic externalities, including congestion, accidents, use of energy and emissions. For instance, about 42,500 people are killed and 3,500,000 injured every year due to road traffic crashes in the European Union (ETSC, 1999). In addition, increasing traffic congestion and related environmental stress is expected in the coming decades (e.g. OECD, 2000). To a large extent, these problems can be attributed to improper or suboptimal

* Correspondence to: V. A. W. J. Marchau, Faculty of Technology, Policy and Management, Delft University of Technology, PO Box 5015, 2600 GA Delft, The Netherlands. E-mail: vincentm@tbm.tudelft.nl

Copyright @ 2003 John Wiley \& Sons, Ltd. 
driving behaviour. For instance, regarding traffic safety, Smiley and Brookhuis (1987) estimate that some $90 \%$ of all traffic accidents can be attributed to human failure. Congestion is the result of the drivers' trip-making decisions (departure time, route, etc.) and minute-by-minute vehicledriving behaviour (e.g. distance keeping, lane changing) (Lindsey and Verhoef, 2000). To improve vehicle-driving behaviour, various technological systems are developed and implemented, which undertake actions in case of improper or suboptimal driving behaviour. Known as Automated Vehicle Guidance (AVG) systems, they automate, to a certain degree, the driver's acceleration, braking, and steering tasks.

The range of possible AVG systems is wide, varying from systems supporting the driver in one specific driving task (e.g. proper distance keeping, blind spot obstacle warning, lane keeping) up to highly advanced systems where the driver's steering, throttling and braking tasks are fully automated (e.g. the auto-pilot). The technological feasibility of most AVG systems is no longer the main issue, as has been demonstrated in several experiments and pilots. These experiments have shown that AVG systems hold potential for improving road traffic performance significantly. For instance, it has been estimated that large-scale implementation of collision-avoidance systems, supporting the driver in the case of imminent crash danger with oncoming vehicles or obstacles, could reduce road fatalities up to $45 \%$ (e.g. Hiramatsu et al., 1997; Sala et al., 1997). The largescale implementation of systems supporting the driver in keeping a proper distance from the nearest vehicle ahead (adaptive cruise control) could increase road capacity up to $25 \%$, depending on system parameters (e.g. Minderhoud, 1999). Eventually, these developments could end up with the full taking-over of driving tasks, allowing for feet-off and hands-off driving. This 'auto-pilot' is likely to be implemented first on dedicated lanes on major highways, enabling equipped vehicles to group themselves into 'platoons'. Preliminary studies show that fully automated driving lanes, compared to manual driving lanes, could generate $80-90 \%$ less accidents and a $200-300 \%$ higher level of capacity use (Kanaris et al., 1997). Together with substantial reductions in travel time (50\%) and fuel consumption and emissions due to better traffic throughput, this implies high potential individual and societal advantages.

In view of the above, transport policy makers are increasingly interested in the automation of vehicle driving tasks, as this could contribute significantly to general transport policy goals, including (e.g. Ministry of Transport, 2001; CEC, 2001): an improvement in accessibility (reduction of congestion, more efficient use of road infrastructure); an improvement in traffic safety (reduction of accident fatalities and injuries) and reduction of environmental deterioration (less energy use and emissions, less consumption of scarce space). However, current policy developments regarding AVG are highly complicated by uncertainties regarding future AVG technologies and their implementation (Van Geenhuizen and Thissen, 2002). These uncertainties stem, for instance, from (Marchau, 2000):

- The lack of an univocal specification of how AVG technology implementation will proceed in terms of functionality, user groups and types of roads to be used, etc.

- The alignment of AVG implementation with general transport policy goals (i.e. most AVG system performance has been assessed under rigidly controlled experimental conditions with limited real-world validity).

- The fulfilment of specific societal conditions in support of AVG implementation (e.g. liability rules, social acceptance, economic feasibility).

Until now, attempts to reduce these uncertainties through research have been strongly technology driven, focusing on the exploration of technical opportunities of specific AVG technologies and/or specific aspects of AVG implementation. Most of the more societal questions have not been studied 
yet (Van der Heijden and Wiethoff, 1999). This could hinder the development of early AVG systems or lead to the implementation of systems, which serve the producers and individual consumers' interests but might conflict with more general transport policies. Therefore, there is a need for broader pictures of AVG implementation which treat possible AVG applications, including their potential impacts on transportation system performance and the enabling, societal conditions for implementation, in an integrated way (Marchau and Walker, 2002).

The key question is how this type of research should be performed. Traditional assessment approaches used by transportation professionals in this field are mostly based on trend extrapolations and quantitative modelling. The resulting models typically focus on the physical part of road transportation, i.e. infrastructure and traffic. In these studies, it is assumed that there is sufficient insight into the complex system to accurately model the systems structure and to predict the future with sufficient accuracy. However, the decisions of both individuals and organizations in transportation processes are hardly or not at all considered, even though these decisions largely determine the impacts of AVG implementation. For instance, in these traditional assessments, the contribution of AVG implementation to general transport policy goals is based on the assumptions that all vehicles are equipped with a perfectly functioning system, and that drivers use the system exactly as it was designed to do. Yet only a very few vehicles have been equipped with AVG facilities, different systems have become available by different suppliers, systems have malfunctioned, and drivers have behaved unexpectedly when using AVG systems. These developments seriously undermine estimated positive impacts. Summarizing, it seems that traditional evaluation approaches cannot cope with such uncertainties (Haynes and Qiangsheng, 1993; Bristow et al., 1997; Stough, 2001).

Recently, innovative methodologies for handling uncertainties in new transport technologies have been suggested (e.g. Gifford and Schwartz, 1995; Moon et al., presentation at the 8th World Conference on Transport Research, Antwerp, 1998; Stough, 2001). These studies involve the development of frameworks for structuring uncertainty on transport, traffic and technologies, and result in methodological recommendations to be taken care of in this context. Although useful at a conceptual level, these studies do not elaborate operational methodologies that could be applied in a straightforward manner to cope with the uncertainties of AVG implementation. Hence, from the research perspective, the further development of such methodologies remains a challenge. In this article, we illustrate how this challenge can be tackled (Marchau, 2000), as part of a larger, recently finished, research program on the future of AVG within the Dutch Research School of Transport, Infrastructure and Logistics (TRAIL). Another three PhD projects in this programme have been studying implementation of AVG at the specific levels of, respectively, driver and travel behaviour, traffic performance and infrastructure design, and legislation and tort liability (for an overview see Van der Heijden and Wiethoff, 1999). The programme was initiated in the mid-1990s in reaction to a strong governmental interest in improving intelligence in traffic and transport management. Since then, the Netherlands has pursued a guiding role in this field, illustrated by extended demonstrations of Automated Vehicle Guidance in 1998. Moreover, the government initiated the set-up of and investments in scientific knowledge transfer organizations, laboratories, pilot projects and public-private research programmes. The research programme referred to in this article was financed by the Delft University of Technology and was meant as a contribution of this university to these public-private initiatives. Consequently, the researchers in the programmes had regular cooperation and discussions with representatives in the field of public transport policy making. Moreover, in recent years the researchers started to participate in various European projects in the context of the Fifth Framework, actively disseminating generated knowledge to automotive industries and other 
research institutes and policy makers. All these initiatives have led to a follow-up research programme with six PhD students financed for four years by the Dutch National Science Foundation, starting in mid-2002.

This paper is organized as follows. The next section explores the field of Technology Assessment (TA) with the aim of developing a methodological approach for coping with uncertainty of future AVG implementation. As choices made for a specific TA methodologies all are typically 'problem driven', the third section gives an in-depth coverage of the problem field, with a systemic view on the relationship between AVG technologies and the transport system. Based on this view, the methodological approach applied in our study is conceptualized in the fourth section. In the fifth section this conceptual approach is specified and applied. In the final section some conclusions are drawn and discussed.

\section{METHODOLOGICAL FRAMEWORK}

The field of interdisciplinary research on the possibilities and consequences of technology implementation in general is Technology Assessment (TA). Hence, this field of research is logically of interest for this study. The origin of TA dates back to the 1950s when technology forecasting studies were performed to support policy decisions on technological investments of large industries and governments. In the 1960s, the awareness of negative impacts of technologies grew and the emphasis of technology forecasting studies shifted towards impact assessment. These TA studies sought to assess the intended as well as the unintended impacts of technological developments on society (Coates and Coates, 1989).

In the 1970s and 1980s, the often implicit assumption that the development of a technology as well as its societal impacts can be predicted was criticized. Practical experience and in-depth research indicated that technological developments are not autonomous but influenced by strong interactions with their social context (Bijker et al., 1987). Forecasts were often wrong or unexpected impacts were observed (e.g. Ascher, 1978). Moreover, TA practice pointed out that decision-making processes regarding innovative technologies are much fuzzier than is often assumed, and that political rationality plays an important role (Smits and Leyten, 1989). Due to this criticism, the focus of TA changed from an objective analysis of future systems towards understanding the role of specific stakeholders in socio-technical decision making. Consequently, TA studies have gradually become more process-oriented, in an attempt to incorporate societal or sectoral aspects, as well as the stakeholders' preferences and actions in the design of new technologies. In this context, the term Technology Foresight combines supporting analyses and communication processes where informed stakeholders participate in a forward-looking exercise (Grupp and Linstone, 1999).

The increased variety of TA studies over the years has had consequences for the methodological approaches used within TAs. Next to traditional technology forecasting and impact assessment, approaches have been developed which focus on stakeholder participation regarding a problem situation and potential technological solutions. Nowadays, the number of methods used for performing technology assessments is both large and diverse. Van den Ende et al. (1998) present a useful structuring of this variety. Based on their overview and other sources, Table I has been constructed. Here, a distinction is made between the assessment methodology and the methods that are applicable within TA. The methods help in studying a specific aspect of the TA problem. The assessment methodologies, in turn, seek to integrate different aspects of the technology and/or related decision process. Taken together, assessment methodologies and methods characterize the 
Table I. A taxonomy of Technology Assessment methodologies and methods

\begin{tabular}{llll}
\hline $\begin{array}{l}\text { TA aim } \\
\text { TA approach }\end{array}$ & \multicolumn{1}{c}{ Analysis } & \multicolumn{1}{c}{ Intervention } & \multicolumn{1}{c}{ Reflection } \\
\hline $\begin{array}{l}\text { Assessment } \\
\text { methodology }\end{array}$ & Technology forecasting & Interactive TA & Structured reasoning \\
& $\begin{array}{l}\text { Backcasting analysis } \\
\text { Impact assessment } \\
\text { Cost-benefit analysis }\end{array}$ & $\begin{array}{l}\text { Consumer CTA } \\
\text { Strategic niche } \\
\text { management }\end{array}$ & Historical case research \\
& Scenario analysis & & \\
& Market analysis & & \\
Assessment & Analogies & Consensus conference & Workshops \\
methods & Monitoring & Interactive workshops & Interviews \\
& Morphological analysis & Gaming & Literature surveys \\
& Trend extrapolation & & \\
& Modelling & & \\
& Policy capture & & \\
\hline
\end{tabular}

methodological approach. A further distinction between assessment methodologies and methods used in TAs can be based on the aims of the study: analysis, intervention, or reflection.

The category of 'analysis' refers to various analytical assessment methodologies and methods in technology assessment studies. These include methods such as forecasting, scenario construction, impact analysis, market analysis, and trend exploration. Overviews and detailed descriptions of these and other methods can be found in several textbooks (e.g. Porter et al., 1991; Martino, 1993). The second category includes intervention methodologies and methods used to intervene in decision processes on technology development and implementation. Typical methods used in these processoriented approaches are consensus conferences, interactive workshops, and gaming. The category of reflective methodologies and methods is used for organizing the process of intervention.

The remaining question is which TA approach(in terms of assessment methodologies and methods) is appropriate for reducing uncertainties of AVG implementation? The answer should be guided by the aims of the technology assessment: analysing technology development and implementation, intervening in technology development and implementation, or identifying the ways how to intervene in technology development and implementation. The aim in our study is to identify and reduce uncertainty regarding the implementation of AVG technology. Hence, in terms of the above-defined classification this points at methodologies and methods for analysis.

In the literature, few criteria have been presented to make choices between different analytical assessment methodologies and methods (Porter, 1995). Most TAs have been developed in practice and few practitioners have explicitly reflected on their methodological choices (Van den Ende et al., 1998). In general, choices for a specific technology assessment study are typically determined by the problem by the assessor and the objectives of study (Langenhove and Berloznik, 1998). Consequently, the TA researcher is forced to make her or his choices within the general framework presented above. In particular we have to answer the following two related questions:

(1) Which TA assessment methodology (for analyses) is appropriate to reduce uncertainty regarding AVG implementation?

(2) Given this methodology, which TA method(s) (for analyses) can be used to perform this reduction? 
The choice of a specific, analytical TA approach depends on either the assumption that future AVG developments are a continuation (i.e. extrapolation) of the past or the assumption that significant technological discontinuities cannot be excluded in advance. In the former case, well-known methodologies and methods are available. If, however, future surprises cannot be excluded, the probable nature of possible discontinuities should be studied in depth. At present, there is not sufficient information to make a well-founded choice between these two assumptions. Therefore, the TA approach should start with an in-depth study of the relationship between AVG technologies and the transport system components to identify the dynamics of future AVG implementation and the underlying causal mechanisms.

\section{UNDERSTANDING THE DYNAMICS OF AVG TECHNOLOGY IMPLEMENTATION}

\section{A structured view of AVG technologies and the transport system}

Current AVG consists of a variety of systems which can be structured by Hall's (1995) general framework. In this framework, AVG is structured based on three principal entities: the vehicle, the driver, and the road infrastructure. Each entity contains five basic devices, which have to cooperate to perform the vehicle-driving task: sensors, intelligence, memory, transmitters, and actuators (see Figure 1). Sensors collect information by observing driving conditions and/or by receiving messages from, for instance, other vehicles, the road infrastructure, road operators, etc. This data is processed by an intelligence device that formulates commands and composes messages, based on a set of interpretation heuristics and decision rules. The knowledge and experience of the entity are stored within the memory. The information from the intelligence is transformed into actions by the actuator and transformed into relevant messages by the transmitter.

Various AVG systems can be constructed as unique combinations of devices with varying capabilities. Sensors can collect data on, for instance, the state of the driver, the state of the vehicle, obstacles in the vicinity of the vehicle, road conditions, traffic conditions, etc. (Komoda and Goudy, 1995). The intelligence and memory constitute the core of the entity as here decisions are made for executing specific actions to attain specific system objectives (e.g. vehicle-following, lane keeping, lane changing). There are various options for both the type of actuator (e.g. throttle, brake and

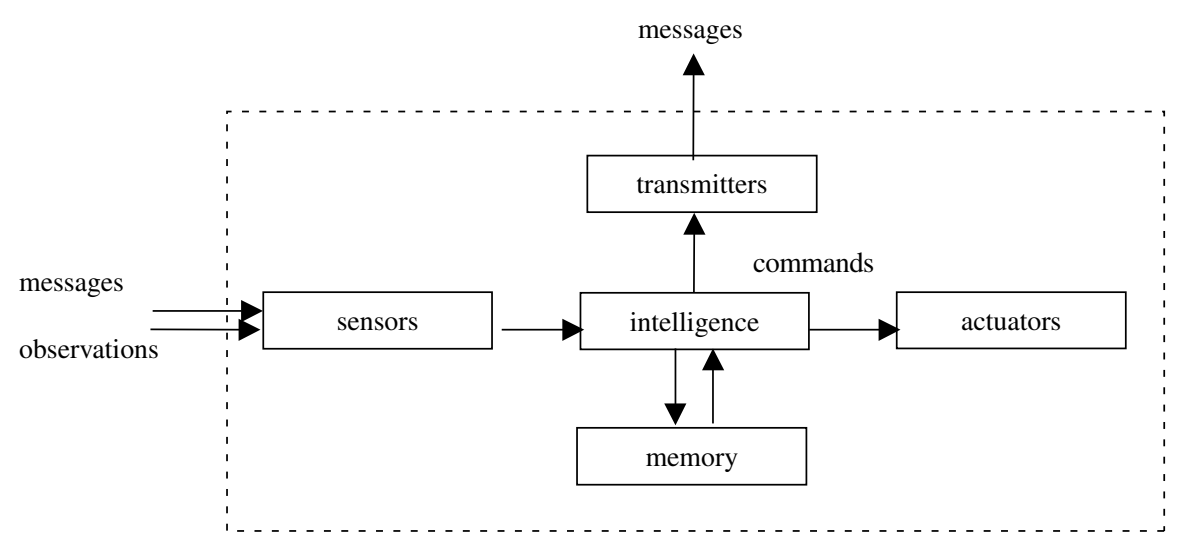

Figure 1. The basic devices of AVG 
steering) and for the level of actuation (e.g. advisory, intervention). With regard to the transmission of messages, one can distinguish between AVG systems that are self-supporting or autonomous and systems which cooperate, i.e. communicate, with other entities. Different combinations of these system elements are currently studied, tested, and improved, in relation to user functions such as: longitudinal collision avoidance, lateral collision avoidance or driver-vehicle-road monitoring. In what follows, we focus on services that are essential for fully automated driving. Thus, systems for longitudinal collision avoidance, lateral collision avoidance and automated highways are of primary interest.

In order to identify the possible future dynamics regarding the implementation of the AVG systems in the transport system, a workable structuring of the transport system is helpful. We used the systemic view for the transport system as presented in Figure 2 (Van der Heijden, 1999). Here, the transport system consists of seven subsystems. Four subsystems comprise the transport system's physical features: infrastructure, vehicles, cargo and passengers and spatial/economic patterns. The other three subsystems are the three markets: the traffic market, the transport service market, and the transport needs market. The traffic market represents the interaction between the vehicle capacity and the supply of infrastructure facilities. The output of this market results in the traffic patterns, the allocation of vehicles to infrastructure networks in time and space. The transport market represents the interaction of the actual transportation demand over space and time and the transportation services provided, resulting in the transport performance or mobility patterns in terms of trips made, modes used and routes chosen within a certain time period. The transport needs market represents the interaction between the latent demands of individuals for performing specific spatially dispersed activities, on the one hand, and the available transport options and the financial and temporal budget, on the other, resulting in an actual transport demand. The potential need for transport results from the spatial, social, economic and temporal organisation of society.

These seven subsystems roughly tell us how major processes in the transport system function. Each subsystem generates its own unique processes and problems and has unique stakeholder interactions; from an analytical point of view, it is therefore valid to regard them as subsystems. From a synthesis point of view (e.g. in policy making), it is important to pay also attention to interactions between the subsystems. A lower subsystem facilitates (supports) the processes within the higher subsystems. A higher subsystem, however, does not support processes in the lower subsystem: it places functional requirements on the supporting facilities and services offered by the lower subsystem.

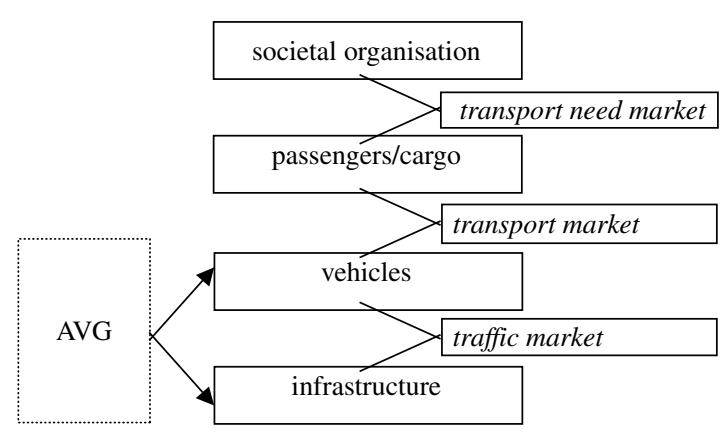

Figure 2. A market structuring of the transport system 
AVG applications intervene at the levels of the vehicle, traffic market and infrastructure components (see Figure 2). The basic challenge is to improve vehicle control and thus optimize the interactions between driver and vehicle. In consequence, it is assumed that AVG applications contribute to a more steady traffic flow and therefore also to a more efficient use of capacity, increased comfort, and reduced fuel consumption.

\section{Possible dynamics of AVG implementation}

In the above-described systemic framework, the possible dynamics of the implementation of the AVG technologies within the transport system can be analysed. The degree of dynamics refers to the range and sequence of future states of the different transport subsystems when AVG applications are implemented. This degree of dynamics has been explored by collecting the opinions of Dutch experts, supplemented with a scan of the (international) literature (Marchau and Van der Heijden, 1997). It resulted in the following findings:

- Infrastructure level: there are various infrastructure adaptations which could facilitate the traffic system in relation to AVG systems. For instance, the transmission of information about actual infrastructure conditions (e.g. rain, snow) to in-vehicle speed adaptation devices could increase traffic safety and efficiency significantly. Next, most AVG applications are developed for use on motorways or dedicated lanes. However, the traffic situation at these road types is relatively easy as compared to, for instance, urban and rural roads: the traffic is relatively homogeneous, the roadway curvature is limited and the road boundaries are clearly set. Consequently, supporting vehicle control seems the least complex challenge for this road network as compared to other road networks.

- Traffic market level: it is not clear how AVG will affect traffic flow behaviour and therefore indirectly traffic safety and road efficiency (e.g. Marsden et al., 2001). Although, in general, AVG intends to improve traffic efficiency (or at least not to reduce road capacity), there are studies which forecast (small) capacity decreases for a higher penetration level within mixed traffic, specific settings of some systems and dense traffic conditions (Minderhoud, 1999). Furthermore, intended safety benefits could be counteracted by behavioural adaptation of drivers (Endsley and Kiris, 1995). These negative efficiency- and safety impacts might increase in a mixed situation of vehicles with different systems, each having its specific type of support and performance.

- Vehicle level: the question of which specific in-vehicle systems will significantly improve the vehicle driving performance is still open. The functionality of systems can vary considerably, depending on varying capabilities and design parameters of AVG system elements. Regarding collision-avoidance systems, for instance, a wide range of available technologies is used to build potential countermeasures related to different crash types (Wassim, 1994). Furthermore, for each specific crash type several sensing options, decision-making algorithms, and types of control are possible. Finally, communication between vehicles and/or between vehicles and the road infrastructure could improve AVG operating performance in terms of less complex sensor requirements and reductions of the occurrence of false or nuisance alarms (Shladover, 1997).

- Transport market level: the implementation of AVG could influence existing travel behaviour negatively in terms of a modal shift and route choices in favour of AVG-equipped vehicles. For instance, when AVG-equipped vehicles become safer and more comfortable, car usage could become more attractive than public transport (Nijkamp et al., 1996). Consequently, more vehicle trips might be undertaken, counteracting initial safety (and efficiency) benefits. Route choices could be influenced, for instance, by the fact that a system is only applicable on a certain type 
of road because of required intelligence in the infrastructure. Hence, the long-term influence of AVG on travel behaviour might countermeasure short-term traffic impacts.

- User level: there are several user groups for which AVG technologies could become available, as different systems providers might focus on different market segments. Collision-warning systems and adaptive cruise controls are currently available for specific groups (heavy vehicles, luxury cars). The speed of technology adoption by other groups is uncertain. For instance, in the Netherlands antilock braking systems are rapidly becoming common features of vehicles. This could also be the case for AVG systems.

- Transport needs market level: the implementation of AVG systems could influence activity patterns in the longer run. As choices with respect to e.g. locations for living, working, shopping or recreation are also based on their qualitative accessibility, new patterns of activity could originate from the significant transport quality improvements to be induced by these systems. It has been found, for instance, that fully automated facilities have the potential to influence land use patterns significantly (Stevens et al., 1995). For instance, residential and business areas could become more decentralized if automated driving appears to reduce travel time.

- Societal organization level: AVG systems could easily generate new transport needs. For example, elderly and disabled people could be attracted to more car trips if their driving abilities are improved by these systems. Freight operators and public transport operators could be interested in starting or expanding road transport activities due to AVG-induced improved traffic performance. In the Netherlands, for instance, innovative AVG concepts for freight transport are under development, while automated people movers for public transport are already reality in certain places.

These developments are relevant to public policy on AVG. Other factors can be identified which may influence the implementation of AVG (e.g. transport sector contextual changes in economic, environmental, social and legal fields). The lesson is that future, large-scale implementation of AVG could develop in various ways, and as such is highly dynamic.

The implementation of AVG in one specific subsystem may influence processes within other subsystems. Due to dynamic feedback and (sometimes) autonomous mechanisms, future AVG performance can only be analysed in-depth at present, under strict, often disputable, assumptions. Consequently, robust, ex ante evaluations of implementation of AVG are difficult and precise policy strategy development is not yet possible. The development of policy strategies requires, at least, answers to the following societal questions:

- Which AVG systems should (given general transport policy goals) or will (given AVG technological developments) be implemented?

- How should (given general transport policy goals) or will (given AVG technological developments) AVG systems be implemented?

These questions are strongly interrelated and allow for a wide range of answers. Therefore, the choice and the specification of an adequate TA approach require an explicit attention to these ranges and their relationships. In short, there is a need for an approach that generates knowledge on:

- AVG system performance and impacts of large-scale AVG implementation in the transport system, in relation to

- The preferences and choices of stakeholders involved in or affected by AVG implementation.

Such an approach will be described in the next section. 


\section{CONCEPTUAL APPROACH}

In view of the methodological framework above, first an analytical assessment methodology for the TA study should be operationalized based on assumptions about the future character of AVG technology development. The idea of a discontinuous development cannot be eliminated, partly because the evolutionary view (according to which the mechanisms that shaped the historical development of the technology structure its future development) needs to be relaxed. Thus, the application of analytical methodologies based on extrapolations from the past may not be valid.

A methodology which explicitly addresses the assumption of the applicability of extrapolating is backcasting (Robinson, 1988). This involves the identification of a desirable future and stimulates the development of certain innovation processes to reach this desired future. Backcasting aims at identifying conditions for different future paths that can be followed (Mulder, 1995). Whereas forecasting attempts to elicit the most likely future under specified conditions, backcasting tries to identify the degree of freedom of action with respect to possible futures (Robinson, 1990). In backcasting, first an image is identified which might represent a future solution for the societal problem at hand. If such an image can be made explicit, the next step is to identify and assess a path between that future image and today. If such an image does not exist, the path will not be explored and the image will be adjusted (Hojer, 1998).

Once an assessment methodology has been chosen, choices among methods for the assessment methodology are called for. Our investigation into methods for backcasting was disappointing as exclusive methods for backcasting were not found. This was later confirmed by Dreborg (1996). Hence, a combination of conventional methods should be applied in this context. Choices among methods should be based on criteria which account for the contents of the study object and the basic ideas of backcasting. In this study, we adopted the following guiding principles according to which appropriate TA assessment methods should be chosen:

- To start from a broad, holistic view on AVG and the transport system working towards more detailed, operating images

- To start from long-term, societal desired developments of AVG, working backwards to feasible applications in the near future

- To start with analysing technological possibilities, explicitly exploring the implications for the organization of AVG implementation processes.

These principles resulted in the following steps and methods. The first step involves the construction and exploration of alternative AVG concepts for the long term. The main purpose of this step is to structure and limit the set of alternative AVG concepts. A general structuring method being helpful in this context is morphological analysis (Van Doorn and Van Vught, 1978). Applied to our study, it allows AVG concepts to be interpreted as combinations of particular values of AVG basic features. The unique combinations of possible values along the different features produce a variety of alternative concepts. Next, these concepts are evaluated to prune highly unlikely or unfeasible concepts. This involves, for instance, concepts which are unlikely to contribute to general transportation goals (accessibility, safety, and environmental sustainability) and/or concepts which are unfeasible from a technical and/or societal point of view. The elimination of these concepts will limit further research to a set of plausible AVG concepts.

The second step involves an in-depth analysis of the selected set of plausible AVG concepts, in relation to the basic conditions postulated for each concept. This implies identifying and evaluating the initial market characteristics of the different AVG concepts and the barriers that might obstruct 
market introduction and/or further deployment. The expertise and insights of experts are considered appropriate for this type of analysis, as empirical data is lacking and extrapolation from past observations would be an invalid approach. Therefore, the use of methods to collect expert opinions seems to be valuable in supporting this analysis. This analysis will further limit the set of plausible AVG concepts to a set of so-called promising AVG concepts.

The fulfilment of the conditions identified in the previous step depends on a sequence of actions and decisions. Therefore, the third step focuses on the question of whether it is likely that the related decisions and/or actions will be taken in time. This requires insight into the future preferences and choice behaviour of stakeholders. Since this behaviour is not directly measurable (the AVG systems are not available yet), an indirect way has to be followed. A well-accepted approach is measuring preferences, assuming a direct relationship between preferences and (future) decision behaviour. The measurement of preferences of stakeholders regarding (the features of) promising AVG concepts will give us information on potential acceptance. Therefore, such an analysis will further limit the set of promising AVG concepts to a set of accepted concepts.

\section{SPECIFYING AND APPLYING THE CONCEPTUAL APPROACH}

To apply the conceptual approach, the methods which have been used within this approach had to be further specified and operationalized. We highlight the main choices that were made in this context as well as the motivation for these choices. An overview of the different steps, the methods used and their results are presented in Table II.

\section{The construction and exploration of alternative AVG concepts}

In the first step, morphological analysis was applied. In general, morphological analysis converts a system concept under study in terms of basic attributes and their possible values (e.g. Twiss, 1992). Next, all combinations of values are evaluated in order to select the most promising alternatives. The identification of basic attributes is typically related to the purpose for which the concepts are used. The number of attributes to be included depends on the trade-offs made by the researcher: too many attributes complicate the construction and presentation of concepts and too few may give an inadequate or only global description of concepts. The specification of values of the attributes primarily depends upon the nature of the attributes. Some only have two or three values, as for other attributes, the number of values might be significantly higher. However, an increasing number of values rapidly increases the number of alternative concepts to evaluate. For instance, four attributes with four values each gives $4^{4}=256$ alternatives. It is difficult to analyse such large sets of

Table II. Conceptual TA approach of this study

\begin{tabular}{|c|c|c|}
\hline Steps & Method & Results \\
\hline $\begin{array}{l}\text { 1. Construction and exploration of alternative } \\
\text { AVG concepts }\end{array}$ & $\begin{array}{l}\text { Morphological } \\
\text { analysis }\end{array}$ & $\begin{array}{l}\text { A set of plausible } \\
\text { AVG concepts }\end{array}$ \\
\hline $\begin{array}{l}\text { 2. Analysis of the conditions for the } \\
\text { implementation of plausible concepts }\end{array}$ & Delphi method & $\begin{array}{l}\text { A set of promising } \\
\text { AVG concepts }\end{array}$ \\
\hline $\begin{array}{l}\text { 3. Analysis whether conditions for promising } \\
\text { concepts will be fulfilled in time by crucial } \\
\text { stakeholders }\end{array}$ & $\begin{array}{l}\text { Stated preference } \\
\text { modelling }\end{array}$ & $\begin{array}{l}\text { A set of accepted } \\
\text { AVG concepts }\end{array}$ \\
\hline
\end{tabular}


alternatives. In our study, the system view on AVG and the transport system as presented above has been used to facilitate the selection process of attributes and values. This system view gives an aggregate, comprehensive picture of future AVG within the transport system, in terms of physical components, their interaction via markets and the impacts of decisions within various markets. The selected attributes and their values are presented in Table III.

The set of possible AVG concepts were defined as the set of all possible combinations of values of key attributes. There are $3^{4} \times 2=162$ of such combinations. In theory, each combination could be regarded as an alternative; but an in-depth study of each of the 162 AVG concepts would not have been manageable in developing a robust policy framework. There was a need to reduce this set. This could be achieved by eliminating those concepts, which are unlikely and/or impossible to implement. Unlikely primarily referred to the potential contribution of the AVG concept to general transportation goals, i.e. accessibility, safety, and environmental sustainability; impossible referred to the technical and societal feasibility of implementing an AVG concept on a large scale within the time horizon of this study. The concepts which did not meet at least one of these criteria or for which this was highly uncertain are labelled implausible. The result of this elimination process was defined as a set of plausible AVG concepts. Due to the systematic derivation of AVG concepts from basic attributes, it was assumed that an AVG concept was implausible where one of the combinations of levels of constituting attributes was implausible. The implausibility of a partial combination was judged on the basis of expert knowledge expressed in the literature. Several partial combinations of AVG concepts were found logically impossible and/or unlikely. Based on this procedure the set of future possible AVG concepts was reduced to manageable proportions, enabling for further, in-depth research on the remaining concepts. By further application of a temporal dimension, seven bundles of promising, evolving AVG concepts could be defined. Basically, the future implementation of these concepts was assumed to depend upon the assumption one holds on the nature of future AVG developments. For instance, assuming that the implementation of these concepts would follow an evolutionary pattern implies that there would probably first be informing and assisting intelligence in vehicles, either for longitudinal or lateral support, functioning together with conventional vehicles and applicable on motorways only. If a more revolutionary development is assumed, the development could start with the implementation of fully automated devices in vehicles and infrastructure, for both longitudinal and lateral support, functioning on dedicated lanes for target groups only.

\section{Evaluating basic conditions for the implementation of AVG concepts}

The next step involved a more in-depth analysis of the selected set of plausible AVG concepts, in relation to the basic conditions postulated for each of the concepts. This implied identifying and evaluating the initial market characteristics of the plausible AVG concepts and the barriers that

Table III. Key attributes and values of AVG concepts

\begin{tabular}{llll}
\hline Attributes of & \multicolumn{2}{c}{ Values } \\
\cline { 2 - 4 } AVG concept & \multicolumn{1}{c}{$(1)$} & \multicolumn{1}{c}{$(2)$} & \multicolumn{1}{c}{$(3)$} \\
\hline Function & Longitudinal & Lateral & Longitudinal and lateral \\
Automation & Information & Assistance & Automated \\
Instrumentation & Vehicle & Vehicle/infrastructure & Infrastructure \\
Users & Target groups & All users & All roads \\
Roads & Dedicated lanes & Motorways & \\
\hline
\end{tabular}


might obstruct market introduction and/or further deployment. As stated in the previous section, expert knowledge was considered appropriate for this type of analysis. In particular, the Delphi method was applied in this context because of its structuring ability of communication and its typical feedback properties over different interrogation rounds. These method characteristics were considered very important for this type of exploratory research (Rowe et al., 1991). In general, the Delphi method consists of a series of repeated interrogations or rounds of experts. After the initial interrogation, each subsequent interrogation is accompanied by information about the preceding round of responses, usually presented anonymously by some group statistic. The experts are thus encouraged to reconsider and, if appropriate, to change their earlier response in the light of replies of other experts. As soon as a desired level of consensus has been reached, the final group position is determined by calculating some measure of central tendency. The strength of the Delphi method is its ability to explore issues that require personal judgement (Gordon, 1994). There are, however, some important points which should be considered in designing a Delphi study. First, the reliability and accuracy of the results might be limited. Second, the selection of experts should be proper to avoid a 'garbage in garbage out' effect. Finally, a false idea of consensus might occur when the predefined criterion for consensus within the group does not give any information on the consistency of the individual answers over different rounds.

The notion of reliability and accuracy primarily refer to the forecasting function which the Delphi method often serves. The typical forecasting function of the Delphi technique was not prominent in our study. Experts were asked to indicate only the initial market characteristics of the different plausible AVG concepts they expected up to 2020. Most of our study, however, aimed at identifying and estimating the importance of conditions of future implementation of AVG concepts. The next issue involved the selection of experts. In our study, 117 experts were carefully selected worldwide, based on a structured search in a bibliographic database, containing references to all aspects of AVG. Furthermore, each expert was asked to name other experts. Finally, in order to check the expertise, these experts were asked to mention their degree of expertise in each system under consideration. The last point of attention involved the possibility of false consensus. In order to avoid this, the stability of individual expert opinions over subsequent rounds was measured, and used as a stopping criterion. Chaffin and Talley (1980) link consistency of individual judgements to the so-called 'individual stability of answers': the idea that the response frequencies of two subsequent rounds do not differ significantly from each other.

The Delphi method applied was based on a questionnaire with mainly pre-specified answer options, sent to a number of experts who were asked to indicate the time period of market introduction and the user costs for different AVG systems. Furthermore, they had to indicate for which type of user groups and for which specific roads a system will become available at initial market introduction. The next step was to assess the importance of the factors which could obstruct a system's market introduction and/or further developments, using a 5-point scale. Several factors were presented based on a review of the recent literature. Respondents, however, could add missing barriers during the first round of interrogation. Finally, the experts indicated the relative importance of several public policy measures to overcome these barriers.

In the first round of the Delphi study, 117 participants were approached, 65 of which returned a completed questionnaire. Fifty of the 65 questionnaires sent out in the second round were returned. In the third and final round, 40 of 50 questionnaires were returned. These response rates are quite satisfying, compared to other studies described in the literature and given the considerable amount of work asked from the respondents for filling in the questionnaires. The response is further specified 
Table IV. Delphi response over rounds regarding geographical background and affiliation

\begin{tabular}{lcccc}
\hline Background respondents & $\begin{array}{c}\text { Questionnaires } \\
\text { sent out }\end{array}$ & $\begin{array}{c}\text { 1st round } \\
\text { response }\end{array}$ & $\begin{array}{c}\text { 2nd round } \\
\text { response }\end{array}$ & $\begin{array}{c}\text { 3rd round } \\
\text { response }\end{array}$ \\
\hline Geographical: & & & & 21 \\
$\quad$ Europe & 58 & 39 & 30 & 12 \\
$\quad$ North America & 42 & 16 & 13 & 7 \\
$\quad$ Japan & 17 & 10 & 7 & 6 \\
Affiliation: & 12 & 7 & 6 & 9 \\
$\quad$ Government & 31 & 16 & 13 & 12 \\
$\quad$ Industry & 35 & 24 & 17 & 40 \\
$\quad$ Research institute/consultancy & 39 & 18 & 14 & 50 \\
$\quad$ University & 117 & 65 & 50 & \\
Total & & & & \\
\hline
\end{tabular}

in Table IV. Overall, the Delphi panel had adequate expertise because each respondent had at least minor expertise on one AVG system addressed in the questionnaire.

The Delphi study suggested that the evolutionary development of AVG is not at all obvious. There still exist many barriers which obstruct successful implementation. Only for the short term, i.e. before 2005, there existed a strong consensus that warning devices for speed headway keeping, lane keeping and obstacle avoidance will become (further) available, next to vehicle-following systems with limited deceleration capabilities. The introduction of more advanced systems taking temporary control of the vehicle in dangerous situations was still considered to be uncertain. Overall, systems are initially expected to be of use on motorways only, and to be adopted by professional drivers and fleet operators. The study further pointed out that the contribution of most support systems to road capacity gains and reduction of environmental impacts are (highly) uncertain. Moreover, safety improvements may be achieved but these are strictly limited to those driving situations for which the system is designed and these could easily be counter-measured by more risky driving behaviour. Furthermore, for each type of support various systems could become available with different operating characteristics. It is unclear how this diversity will affect total traffic performance. Most uncertain seemed the implementation of the auto-pilot, due to various technical as well as non-technical arguments. As the implementation of more advanced AVG systems appeared to be very uncertain, a stronger focus on systems that can be implemented in the short term was required. For the mid-term, i.e. up to 2010, the Delphi study pointed out that the implementation of the first AVG systems will be dominated by market opportunities for these first systems. Therefore, it was important to gain insight into the preferences of the market and the willingness of potential users to purchase such AVG systems.

\section{Market preferences about the implementation of AVG concepts}

In the third step, both drivers and fleet operators of cars, lorries and buses were questioned about their preferences for selected AVG alternatives from the previous step. In earlier studies on user preferences for AVG, the respondents have evaluated different attributes of system(s) separately (e.g. Becker, 1994; Sayer et al., 1995; Kemp et al., 1998; Hoedemaeker, 1999). In these studies attributes have been presented one by one and the respondents have been asked to evaluate each attribute on some measurement scale according to their needs and preferences. This measurement method is relatively easy to construct and administer. Moreover, the responses potentially have 
high 'reliability', i.e. when the evaluation of attributes is repeated later with the same respondents, almost the same results are obtained. However, this approach has serious limitations in terms of predicting overall preference and choice behaviour (e.g. Oppewal, 1995), as individuals tend to over- or underestimate the importance of separate attributes. This might be explained by the fact that usually in real life more than one attribute plays a role so that individuals make trade-offs among the different attributes of an alternative. These trade-offs are not taken into account by traditional measurement approaches.

The trade-offs among attributes are explicitly considered by another measurement approach, the so-called decompositional stated preference approach, also known as conjoint analysis (Louvière, 1988). In this approach, respondents have to indicate their overall preferences for hypothetical profiles, described in terms of a unique combination of levels of attributes; thus, they are forced to make trade-offs among attributes. As profiles are constructed according to the principles of statistical designs, the overall preference can be decomposed in terms of the weights these respondents attach to separate attribute levels. By interpreting these weights in terms of (part-worth) utilities, it is possible to study the relationship between part-worth utilities and overall utilities in a more valid way as compared to a measurement approach where attributes are evaluated separately.

Therefore, in our study, we adopted conjoint analysis to explore this relationship with respect to AVG concepts. Six pre-specified response groups involving both drivers and fleet owners of cars, lorries and buses were questioned (the total response involved 485) about their preferences regarding several promising AVG concepts, resulting from the previous step in our research. Alternatives were characterized in terms of their functional features, different levels of purchase price and varying impacts on travel time and fuel consumption. These attributes were selected because of their likely influence on preference behaviour of potential users.

On average, drivers and fleet operators judged it neither attractive nor unattractive to have early AVG systems in their vehicle(s). However, the study showed that these findings need to be qualified as the preferences vary significantly on the specific system characteristics and the specific subgroups. Overall, technical functions of systems were evaluated to be less important than the costs and benefits of the system. This might be caused, among others, by the fact that most respondents are unfamiliar with the AVG systems in question and hence with their technical attributes as well. Most influential on the attractiveness is price. Systems which negatively influence travel time performance or fuel consumption lowered the overall utility rapidly, as well as do systems which take over control from the driver. With regard to all technical functions, informing devices are preferred to the other levels. This implies, for instance, that warning is preferred to throttle and brake control in distance-keeping support. As to lane-keeping and lane-changing support, warning support is preferred to both no support and steering assistance. Considering different markets, it was found that lorry drivers, car fleet operators and business drivers consider AVG systems more attractive as compared to the other groups. Hence, these groups might be considered initial target groups for implementation in this decade.

\section{CONCLUSIONS AND DISCUSSION}

In this study, an innovative TA approach was developed and applied in order to identify and reduce uncertainties on future AVG development and implementation. In this context, the field of TA was of interest as it focuses on how the course of future technology developments can be directed in 
societal desired ways. It appeared that the field of TA is characterized by a large variety in terms of problem areas applied, the objectives of the TA, the methodologies used for study, and the methods used within the TA. This variety is an advantage in the sense that TA offers a broad, flexible framework for further choices related to the specific subject of study. It is, however, a disadvantage in the sense that most TA studies are consequently typically problem-driven. Evident scientific criteria for choices are consequently lacking in this context and the researcher is forced to make more or less subjective choices. When evident criteria for choices are not available, the logical research strategy to follow is to first structure and limit the subject of study. Otherwise, the degrees of freedom would be too numerous to set up a TA study and to effectively choose and apply research methods.

In this paper, the issue of AVG implementation has been structured and limited using a system view on AVG and the transport system. Based on this view, the need for more in-depth knowledge linking information on AVG performance and public strategy development was identified. A systematic approach has been specified, focusing on particular AVG concepts and the willingness of stakeholders to support the implementation of these concepts. The use of backcasting analysis resulted in the elimination of implausible, unpromising and unaccepted AVG concepts and helped to reduce the scope of analysis to the most viable ones. This approach developed differs from most other TA exercises in the field of AVG (and transport technologies in general). Most previous studies roughly follow the approach from studying different aspects of sophisticated AVG systems in depth (e.g. traffic impacts, driver behaviour, cost-benefits, etc.). Only incidentally do they choose a broader focus. Our study explicitly started from a broader view, incorporating all possible aspects relevant for implementation. Most AVG studies focus on forecasting specific outcomes of AVG implementation, i.e. what will happen in the future. Our study is not focused on forecasting. It involves structuring and setting the agenda for decision-making, i.e. what should be done now, aiming at specifying robust policies regarding future AVG implementation. As such our research approach serves the typical 'direction setting', 'determining priorities', and 'anticipatory intelligence' functions of Technology Foresight in general (Martin and Irvine, 1989). Each approach has its advantages and disadvantages.

An approach focusing on the in-depth study of details logically results in more, often quantified, findings on each aspect studied. This is related to the fact that one can often rely on a set of wellproven sophisticated, research methodologies and methods. However, due to the isolated treatment of the aspects, the real-world validity of the research findings remains disputable. Furthermore, some aspects might be overlooked as they are of no interest to present studies or cannot be studied by the available methods.

An attempt to avoid these disadvantages is made by means of the approach as described in this article. Trying to identify and assess all aspects which might be relevant, based on an overall view, however, hardly enable the assessment of each aspect as thoroughly as in the first approach. Moreover, as stated earlier, there is no specific protocol on how to perform such an analysis or how to choose the set of methods adequate for this type of study. One is forced to use the more traditional TA methods, although these methods have often been developed for other purposes, and/or to make use of methodologies as developed in other research fields. The conclusion must be that our approach should be considered complementary to traditional TA exercises undertaken in the field of AVG.

Ideally, if there are many uncertainties with regard to technological progress and the organizational processes of implementation, one should perform a TA study using both approaches. This 
would help to produce and integrate further insights into all the relevant aspects of technology implementation. Such a complementary approach has been adopted, for instance, in the earlier-mentioned research programme 'Technology Assessment on Automated Vehicle Guidance', of which our study formed part.

The combination of research methods used within this study generated some methodological insights that might be of interest for similar studies. First, morphological analysis, for example, is particularly useful in the phase of problem exploration. However, this requires some prior knowledge about the technology that is being studied, as there is a need to appropriately identify the key attributes and their possible values in the alternative concepts. Furthermore, the plausibility of alternatives has to be judged carefully. Concepts could be initially excluded which, after all, might become plausible due to accelerated technological progress, for instance.

Second, most (dis)advantages of the Delphi method relate to the aims and set-up of the study. If the Delphi method is used to forecast the implementation of future technologies, requiring some level of consensus among experts, the results may be disappointing. Instead, it may be more fruitful to elicit experts' opinions in order to identify and evaluate barriers to future technology implementation, as well as to study underlying reasons for differences in opinions. Here, a problem is the limited number of experts in a Delphi study, reducing the possibilities for a statistical analysis of arguments. Furthermore, the degree of consensus at aggregate level may not be a misleading stopping criterion for the number of rounds. Instead one could focus on the individual stability of expert opinions over different rounds.

Third, it was learned that conjoint measurement requires a considerable amount of preparatory research, in particular when this method is used to explore the future behaviour regarding highly innovative products or services. Although the set-up of the conjoint measurement task can be described in clear, consecutive steps, each step involves several operational decisions which the researcher has to take based on empirical experiences with the conjoint method in other fields. However, conjoint measurement has been used mostly to study choice behaviour among alternatives that can be readily imagined; hence, for highly innovative products (such as those like AVG systems), care has to be taken to specify the alternatives clearly and unambiguously. A clear presentation is essential for understanding, distinguishing and judging alternatives in a reliable way, that is, resembling their real-world behaviour as much as possible. Preferably, some visual support (pictures, animations or video) or even prototype products is favoured in this respect.

What is left for the future? We have addressed the problems of lacking scientific knowledge concerning the specification, operationalization and application of TA approaches. Therefore, more reflective research is recommended in the field of TA, in terms of comparative assessments using different methodological approaches. This should provide TA researchers with generic, scientific criteria, which can be used for setting up a TA study, including choosing particular methods. This type of research is currently being conducted within several innovative research programmes of which the authors form part. The first results of these activities have been published elsewhere (Marchau and Brookhuis, 2001; Marchau and Walker 2002).

\section{REFERENCES}

Ascher W. 1978. Forecasting-An appraisal for policy-makers and planners. Johns Hopkins University Press: London. 
Becker S. 1994. Summary of experience with autonomous intelligent cruise control (AICC), Part 2, Results and conclusions. In Proceedings of the First World Congress on Applications of Transport Telematics and IVHS. Artech House: Boston, MA; 1836-1843.

Bijker WE, Hughes P, Pinch T. 1987. The Social Construction of Technological Systems. MIT Press: Cambridge, MA.

Bristow AL, Pearman AD, Shires JD. 1997. An assessment of advanced transport telematics evaluation procedures. Transport Reviews 17(3): 177-205.

Chaffin WW, Talley WK. 1980. Individual stability in Delphi studies. Technological Forecasting and Social Change 16: 67-73.

Coates VT, Coates JF. 1989. Making technology assessment an effective tool to influence policy. In Policy Through Impact Assessment: Institutionalized Analysis as a Policy Strategy, Bartlett RV (ed.). Greenwood Press: New York; 17-25.

Commission of the European Communities. 2001. European transport policy for 2010: time to decide. COM.2001.370, Office for Official Publications of the EC: Luxemburg.

van Doorn J, van Vught F. 1978. Forecasting: Methoden en technieken voor toekomstonderzoek. Van Gorcum: Assen (in Dutch).

Dreborg KH. 1996. Essence of backcasting. Futures 28(9): 813-828.

van den Ende J, Mulder K, Knot M, Moors E, Vergragt P. 1998. Traditional and modern technology assessment: towards a toolkit. Technological Forecasting and Social Change 58: 5-21.

Endsley MR, Kiris OE. 1995. The out-of-the-loop performance problem and the level of control in automation. Human Factors 37(2): 381-394.

ETSC. 1999. Intelligent Transportation Systems and Road Safety. European Transport Safety Council: Brussels. van Geenhuizen M, Thissen W. 2002. Uncertainty and intelligent transport systems: implications for policy. The International Journal of Technology, Policy and Management 2(1): 5-19.

Gifford JL, Schwartz D. 1995. Uncertainty in assessing benefits, cost and financial feasibility of ITS projects, programs and services. In Proceedings of the Second World Congress on Intelligent Transport Systems. VERTIS: Tokyo; 2434-2439.

Gordon ThJ. 1994. The Delphi method. In Futures Research Methodology, Glenn JC (ed.). American Council for UNU-The Millennium Project: Washington, DC.

Grupp H, Linstone H. 1999. National technology foresight activities around the globe-resurrection and new paradigms. Technological Forecasting and Social Change 60: 85-94.

Hall RW. 1995. The architecture of transportation systems. Transportation Research-C 3(3): 129-145.

Haynes K, Qiangsheng L. 1993. Policy analysis and uncertainty: lessons from IVHS transportation development process. Computers, Environment and Urban Systems 17: 1-14.

van der Heijden RECM. 1999. Automation of driver support: introduction to the research challenges. In Automation of Car Driving: Exploring societal impacts and conditions, van der Heijden RECM, Wiethoff M (eds). Delft University Press: Delft; 1-24.

van der Heijden RECM, Wiethoff M (eds). 1999. Automation of Car-driving: exploring societal impacts and conditions. Delft University Press: Delft.

Hiramatsu K, Satoh K, Matsukawa F. 1997. Estimation of the number of fatal accidents reduced by Advanced Safety Vehicle. ASV. Technologies. In Proceedings of the 4th World Congress on ITS. ITS Congress Association: Brussels.

Hoedemaeker M. 1999. Driving with Intelligent Vehicles. Driving behaviour with Adaptive Cruise Control and the acceptance by individual drivers. Delft University Press: Delft.

Hojer M. 1998. Transport telematics in urban systems — a backcasting Delphi Study. Transportation ResearchD 3(6): 445-463.

Kanaris A, Ioannou PA, Ho PS. 1997. Spacing and capacity evaluations for different AHS concepts. In Automated Highway Systems, Ioannou PA (ed.). Plenum Press: New York; 125-167.

Kemp MA, Lapping JE, Kiefer MR. 1998. Consumer acceptance of automotive crash avoidance devices: the results of focus group research. In Proceedings of the 8th ITS America Meeting. ITS America: Washington DC.

Komoda N, Goudy RW. 1995. The standardisation activities in ISO/TC204/WG14 vehicle/ roadway warning and control systems. In Proceedings of the Second World Congress on Intelligent Transportation Systems. VERTIS: Tokyo; 2632-2637. 
van Langenhove L, Berloznik R. 1998. Technology assessment and forecasting for sustainable development. Technological Forecasting and Social Change 58: 1-4.

Lindsey R, Verhoef E. 2000. Congestion modelling. In Handbook of Transport Modelling, Hensher DA, Button KJ (eds). Elsevier Science Ltd: Oxford; 353-373.

Louvière JJ. 1988. Analyzing Decision Making: Metric Conjoint Analysis. Sage Publications: Beverly Hills, CA.

Marchau VAWJ. 2000. Technology Assessment of Automated Vehicle Guidance: Prospects for automated driving implementation. Delft University Press: Delft.

Marchau VAWJ, Brookhuis KA. 2001. Knowledge needs on the implementation of automated driver assistance systems. The European Journal of Transport and Infrastructure Research 1(3): 221-224.

Marchau VAWJ, van der Heijden RECM. 1997. Possible roles of stakeholders towards developments of advanced vehicle control systems. In Proceedings of 30th ISATA Conference, Automotive Automation Ltd: Croydon; 345-352.

Marchau VAWJ, Walker W. 2002. Handling uncertainty on ITS implementation. The International Journal of Technology, Policy and Management 2(1): 1-4.

Marsden G, McDonald M, Brackstone M. 2001. Towards an understanding of adaptive cruise control. Transportation Research-C 9(1): 33-51.

Martin BR, Irvine J. 1989. Research Foresight: Priority-setting in Science. Pinter Publishers: London.

Martino JP. 1993. Technological Forecasting for Decision Making. McGraw-Hill: New York.

Minderhoud MM. 1999. Supported Driving: Impacts on Motorway Traffic Flow. Delft University Press: Delft.

Ministry of Transport, Water Management and Public Works. 2001. The National Traffic and Transport Plan. The Hague.

Mulder H. 1995. Back to Our Future. Physical constraints on sustainable development paths in an energy-based backcasting approach. University of Groningen: Groningen.

Nijkamp P, Pepping G, Banister D. 1996. Telematics and Transport Behaviour. Springer-Verlag: Berlin.

OECD Road Transport Research. 2000. Outlook 2000-Perspectives 2000. OECD: Paris.

Oppewal H. 1995. Conjoint Experiments and Retail Planning: modelling consumer choice of shopping centre and retailer reactive behaviour. TU Eindhoven: Eindhoven.

Porter AL. 1995. Technology assessment. Impact Assessment 13(2): 135-151.

Porter AL, Roper AT, Mason TW, Rossini FA, Banks J. 1991. Forecasting and Management of Technology. John Wiley: New York.

Robinson JB. 1988. Unlearning and backcasting: rethinking some of the questions we ask about the future. Technological Forecasting and Social Change 33: 325-338.

Robinson JB. 1990. Futures under glass - A recipe for people who hate to predict. Futures October: 820-842.

Rowe G, Wright G, Bolger F. 1991. Delphi, a revaluation of research and theory. Technological Forecasting and Social Change 53: 235-251.

Sala G, Clarke N, Carrea P, Mussone L. 1997. Expected impacts of anti-collision assist applications. In Proceedings of the 4th World Congress on ITS. ITS Congress Association: Brussels.

Sayer JR, Mefford ML, Fancher P. 1995. Consumer acceptance of adaptive cruise control following experience with a prototype system. In Proceedings of the Human Factors and Ergonomics Society 39th annual meeting, Vol. 2, Human Factors and Ergonomics Society: 1092-1096.

Shladover SE. 1997. Cooperative advanced vehicle control and safety systems (AVCSS). In Proceedings of the 4th World Congress on ITS. ITS Congress Association: Brussels.

Smiley A, Brookhuis KA. 1987. Alcohol, drugs and traffic safety. In Road Users and Traffic Safety, Rothengatter JA, de Bruin RA (eds). Van Gorcum: Assen; 83-105.

Smits R, Leyten J. 1988. Key issues in the institutionalisation of technology assessment. Development of technology assessment in five European countries and the USA. Futures February: 19-36.

Stevens WB, Harding J, Lay R, McHale G. 1995. Summary and Assessment of Findings from the Precursor Analyses of Automated Highway Systems. The MITRE Corporation: McLean.

Stough RR. 2001. Intelligent Transport Systems - Cases and Policies. Edward Elgar: Cheltenham, UK.

Twiss BC. 1992. Forecasting for Technologists and Engineers; a Practical Guide for Better Decisions. Peregrinus: London.

Wassim GN. 1994. A review of IVHS crash avoidance technologies. In Proceedings of the Workshop: Collision Avoidance Systems - Issues and Opportunities. ITS-America: Washington, DC; 103-124. 
276 V. A. W. J. Marchau and R. E. C. M. van der Heijden

Authors' addresses:

V. A. W. J. Marchaw, Faculty of Technology, Policy and Management, Delft University of Technology, PO Box 5015, 2600 GA Delft, The Netherlands.

R. E. C. M. Van der Heijden, Nijmegen School of Management, Nijmegen University, Nijmegen, The Netherlands. 